\title{
Exploring the Performance Tradeoffs among Stability-Oriented Routing Protocols for Mobile Ad hoc Networks
}

\author{
Natarajan Meghanathan \\ Dept. of Computer Science, Jackson State University \\ 1400 John R. Lynch Street, Jackson, MS 39217, USA \\ Tel: 1-601-979-3661Ｅ-mail: natarajan.meghanathan@jsums.edu
}

Received: July 13, 2010 Accepted: September 7, $2010 \quad$ DOI: 10.5296/npa.v2i3.436

\begin{abstract}
In this paper, we present an extensive ns-2 simulation based performance comparison of three widely known stability-oriented on-demand MANET routing protocols: Associativity-Based Routing (ABR) protocol, Flow-Oriented Routing Protocol (FORP) and Route Assessment Based Routing (RABR) protocol. Our simulations show that FORP routes are more stable than RABR routes, which are more stable than ABR routes. This also results in an increased packet delivery ratio for FORP in comparison to that of RABR and ABR. On the other hand, based on the energy consumed per packet and the average energy used per node, ABR is better than RABR, which is better than FORP. At low network density and mobility, ABR routes incur the lowest delay and as the network density and node mobility increases, RABR incurs lower delay. FORP incurs the highest delay under all conditions. Thus, we see a stability-delay-energy consumption tradeoff among these three stability-oriented routing protocols. Regarding the fairness of node usage, we observe that routes get distributed more evenly with increase in the node mobility and network density. But, still there is an appreciable variation in the energy consumption per node as only the chain of nodes that form stable routes are exhausted to a greater extent.
\end{abstract}

Keywords: Routing Protocols, Stability, Performance Tradeoffs, Mobile Ad hoc Networks, Simulation 


\section{Introduction}

Mobile ad hoc networks (MANETs) are dynamic distributed systems, in which any node can become a source or destination for a communication session and all nodes are considered as peers. The transmission range of the nodes is often limited and hence routes between any two nodes in MANETs are often multi-hop in nature with several intermediate nodes forwarding the data of their peers. Several routing protocols have been proposed in the MANET literature. These protocols select routes either proactively or reactively. Proactive routing protocols attempt to determine routes between any two pairs of nodes, irrespective of their requirement, using periodic exchange of link state updates. Reactive routing protocols tend to determine routes only when required, i.e., in an on-demand fashion, using a broadcast query and reply cycle. Even though, there is a route discovery and maintenance overhead in both classes of protocols, research studies (e.g., [3]) have shown that in dynamic scenarios, typical to that of MANETs, reactive on-demand routing protocols are to be preferred compared to the class of proactive routing protocols.

Based on their route selection principle, routing protocols for mobile ad hoc networks (MANETs) could be classified as belonging to one of the following two categories: minimum-weight based routing (e.g., hop count, delay, energy consumption, etc) and stability-based routing [14][15]. MANET routing protocols are often designed to maintain optimality in one or more performance metrics (like hop count, delay, energy consumption, bandwidth, etc). For example, the Dynamic Source Routing (DSR) protocol [10] instantaneously selects any shortest path that appears to exist and similarly the Ad Hoc On-demand Distance Vector (AODV) protocol [21] chooses the route that propagated the Route Request (RREQ) message with the lowest delay. In dynamic environments like that of MANETs, optimal routes, with respect to a particular metric, keep changing with time. To maintain optimality in their design metrics, routing protocols change their paths frequently and incur a huge network overhead. Hence, we focus on routing protocols which choose routes that can exist as long as possible and the number of route transitions incurred by the routing protocol should be as low as possible. Accordingly, we quantify path stability as the number of route transitions (i.e., route discoveries) a routing protocol should incur for the duration of a source-destination $(s-d)$ session. Quite a significant number of works (e.g., [3][9]) have compared the performance of on-demand non-stability (e.g., minimum-hop) based routing protocols like DSDV [22], DSR [10], AODV [21], TORA [20], etc. Not much has been reported on the performance comparison of stability-oriented MANET routing protocols. This forms our motivation for this paper.

Path stability is an important design criterion to be considered in the development of multi-hop routing protocols for resource-constrained environments like that of MANETs. Wireless links are shared and are limited in their bandwidth. Frequent route discovery attempts could congest the network and also knock out the battery power at critical nodes. The battery reserves at the nodes are to be treated precious as they may be deployed in environments where recharging is next to impossible. Also, nodes in energy-constrained environments like that of the sensor networks and embedded networks cannot afford to lose their battery power quickly. For multi-media applications that require the packets to be 
delivered in-order with negligible variance in the inter-packet delay, frequent changes in the routes traversed by the packets could result in out-of-order packet delivery with high jitter. In the case of reliable data transfer applications, failure to receive an acknowledgement packet within a particular timeout interval can also trigger retransmissions at the source side. As a result, the application layer at the receiver side might be overloaded in handling out-of-order, lost and duplicate packets. Thus, path stability is important from the point of view of Quality of Service too.

A statistical approach for predicting link and path lifetimes has been discussed in [7], and the authors recommend selecting a path that has the highest estimated probability to persist for a required amount of time. But, it is not practical to manually set the various parameters that control the statistical metrics used for path stability. In reality, protocols are often restricted to use quantifiable absolute metrics like link age (indicates how long two nodes have remained as neighbors), residual link lifetime (a measure of how long two nodes will stay together as neighbors from the current instant), signal strength (a measure of the proximity of two nodes), etc to assess link and path stability.

Given the complete knowledge of future topology changes, an efficient, polynomial-time algorithm has been proposed in [17] to determine the sequence of stable routes for a source-destination $(s-d)$ session. In general, the strategy used by the currently available stability-oriented routing protocols to select stable paths is based on the knowledge of the past topology changes and/or prediction of the future topology changes [16]. On this basis, we pick three widely different stability-oriented routing protocols: ABR (based on the knowledge of the past topology changes, i.e., link age), FORP (based on the prediction of future topology changes, i.e., the predicted route expiration time) and RABR (based on the combination of knowledge of the past and prediction of future topology changes i.e., average change in signal strength). ABR uses the degree of association stability, which basically quantifies how long two nodes have stayed together as neighbors, to select paths. FORP selects the route that will have the largest expiration time since the time of its discovery. The expiration time of a route is measured as the minimum of the predicted expiration time of its constituent links. RABR uses the average change in the received signal strength to predict the time when the received signal strength would fall below a critical threshold. A more detailed review of ABR, FORP and RABR is available in Section 2.

We implemented all these three routing protocols in the ns-2 [5] simulator. The performance metrics that are considered to evaluate these protocols are: average packet delivery ratio, average number of route transitions (i.e., route discoveries) per $s$ - $d$ session, average end-to-end delay per delivered packet, average hop count per route, average energy consumed per delivered packet, average energy consumed per node and the standard deviation of the energy consumed per node. As part of the estimation of these values, we evaluate the tradeoffs among these performance metrics. The rest of the paper is organized as follows: In Section 2, we review the ABR, FORP and RABR protocols. In Section 3, we describe our simulation environment and present the simulation results. Section 4 concludes the paper and discusses the future work. 


\section{Review of Stability-Oriented Routing Protocols}

\subsection{Associativity-based Routing (ABR) Protocol}

ABR [24] classifies a link as stable or unstable based on the link age. Each node determines the age of a link with its neighbors based on the number of beacons periodically received from that neighbor. If the number of beacons received from a neighbor is greater than an associativity threshold, $A_{\text {thresh }}$, the link with the neighbor is considered stable; otherwise the link is deemed to be unstable. The value of $A_{t h r e s h}$ between two nodes is $2 r_{t x} / v$, where $v$ is the relative velocity between the two nodes and $r_{t x}$ is the transmission range of a node. For example, consider each wireless node in the network to have a transmission range of $250 \mathrm{~m}$ and each node sends a beacon to announce its presence every one second. Let there be two nodes $\mathrm{A}$ and $\mathrm{B}$ that are initially not within the transmission range of each other. If $\mathrm{A}$ is migrating at $10 \mathrm{~m} / \mathrm{s}$, enters into the transmission range of $\mathrm{B}$, and moves through it diagonally, then both A and B can record at most 50 beacons each. Hence $A_{\text {thresh }}=50$ beacons. If the two nodes $\mathrm{A}$ and $\mathrm{B}$ have exchanged more than 50 beacons during the time of a route discovery process, then the two nodes are considered to have a good chance of being in the vicinity of each other for a longer time even though they are moving. In such a situation, the link A - B is considered to be associatively stable. If the two nodes A and B have exchanged 50 or less beacons at the time of the route discovery process, then the link $\mathrm{A}-\mathrm{B}$ is considered to be associatively unstable.

Route discovery in ABR is accomplished using a broadcast-query and reply cycle. If the source node $s$ does not have a route to the destination $d$, it floods a ROUTE-REQUEST packet throughout the network. Each intermediate node forwards the ROUTE-REQUEST packet to its neighbors after affixing its node id, the beacon count and the association stability for the link to the node from which it received the ROUTE-REQUEST packet. A ROUTE-REQUEST packet for a particular route discovery process is not forwarded by a node more than once. After receiving the first ROUTE-REQUEST packet, the destination waits for a fixed time period to receive multiple ROUTE-REQUESTs through different paths. The destination then selects the path that has the maximum proportion of stable links. If two or more paths have the maximum proportion of stable links, the tie is broken by selecting the minimum hop path. Any further tie is broken by choosing a random path among those minimum hop paths. The destination sends a ROUTE-REPLY packet along the reverse direction of the selected route. Each intermediate node through which the ROUTE-REPLY packet is forwarded marks the route as valid and stores the next hop information in its local routing table.

\subsection{Flow-Oriented Routing Protocol (FORP)}

FORP [23] utilizes the mobility and location information of nodes to approximately predict the expiration time (LET) of a wireless link. The minimum of the LET values of all wireless links on a path is termed as the route expiration time (RET). The route with the maximum value of the RET is selected. Each node is assumed to be able to predict the LET values of each of its links with its neighboring nodes based on the information regarding the current position of the nodes, velocity, the direction of movement, and transmission ranges. 
FORP assumes the availability of location-update mechanisms like GPS (Global Positioning System) [11] to identify the location of nodes and also assumes that the clocks across all nodes are synchronized. In our simulations, the location of a node at a particular instant of time is taken from the mobility trace files.

Given the motion parameters of two neighboring nodes, the duration of time the two nodes will remain neighbors can be predicted as follows: Let two nodes $i$ and $j$ be within the transmission range of each other. Let $\left(x_{i}, y_{i}\right)$ and $\left(x_{j}, y_{j}\right)$ be the co-ordinates of the mobile hosts $i$ and $j$ respectively. Let $v_{i}, v_{j}$ be the velocities and $\Theta_{i}, \Theta_{j}$, where $\left(0 \leq \Theta_{i}, \Theta_{j}<2 \pi\right)$ indicate the direction of motion of nodes $i$ and $j$ respectively. The amount of time the two nodes $i$ and $j$ will stay connected, $D_{i-j}$, can be predicted using the following equation:

$$
D_{i-j}=\frac{-(a b+c d)+\sqrt{\left(a^{2}+c^{2}\right) r^{2}-(a d-b c)^{2}}}{a^{2}+c^{2}}
$$

where,

$a=v_{i} \cos \Theta_{i}-v_{j} \cos \Theta_{j}$

$b=x_{i}-x_{j}$

$c=v_{i} \sin \Theta_{i}-v_{j} \sin \Theta_{j}$

$d=y_{i}-y_{j}$

Route request packets are propagated as described before, from the source node $s$ to the destination node $d$. The information recorded in this case by a node $i$ receiving a ROUTE-REQUEST message from a node $j$ is the predicted lifetime of the link $i-j$. The destination $d$ will receive several ROUTE-REQUEST messages with the predicted link lifetimes in the paths traversed being listed. The residual expiration time of a path is the minimum of the predicted lifetimes of its constituent links. The $s-d$ path that has the maximum predicted residual lifetime is then selected. If more than one path has the same maximum predicted residual lifetime, the tie is broken by selecting the shortest (minimum hop path) of such paths.

\subsection{Route-Lifetime Assessment Based Routing (RABR) Protocol}

The RABR protocol [1] functions as follows: The lifetime of a link $i-j$ is predicted using a metric called the "affinity," $a_{i j}$, and it is a measure of the time taken by node $i$ to move out of the range of node $j$. Nodes exchange beacons periodically (for every one second in our simulations). Node $j$ periodically samples (for every $\Delta t$ time units, in our simulations, $\Delta t=1$ second), the strength of the beacon signals received from node $i$. The rate of change of signal strength is given as:

$$
\Delta\left(S_{i j}\right)=\frac{S_{i j}(\text { current })-S_{i j}(\text { prev })}{\Delta t}
$$

The above quantity is then averaged over the last few samples (in our simulations, we consider the last 10 samples) to obtain $\Delta S_{i j}$ (ave). The affinity $a_{i j}$ is then calculated as 
$a_{i j}=$ HIGH (we use 1000) if $\Delta S_{i j}$ (ave) $\geq 0$ (the two nodes are moving towards each other).

$a_{i_{j}}=\frac{S_{\text {thresh }}-S_{i_{j_{(\text {current })}}}}{\Delta S_{i_{j_{(a v e)}}}}$ if $\Delta S_{i j}$ (ave) $<0$ (the two nodes are moving away from each other).

The ROUTE-REQUEST messages are propagated from the source node $s$ to the destination $d$ as in the previous protocols. In RABR, the affinity of the links is recorded in the ROUTE-REQUEST messages and the affinity of a path is the minimum affinity of the constituent links of the path. The destination node chooses the path that has the maximum affinity. If more than one path has the same maximum affinity, the tie is broken by selecting the shortest (minimum hop path) of such paths.

\section{Simulations}

We implemented the ABR, FORP and RABR protocols in the $n s-2$ (version 2.28) simulator [5]. The network dimensions are $1500 \mathrm{~m} \times 300 \mathrm{~m}$. The transmission range of each node is $250 \mathrm{~m}$. We vary the density of the network by conducting simulations with 25 nodes (low density) and 50 nodes (high density). The simulation time is 1000 seconds. All the simulation parameters are listed in Table 1.

Table 1. Simulation Parameters

\begin{tabular}{|c|c|c|}
\hline \multirow{4}{*}{ Simulator } & \multicolumn{2}{|c|}{ ns-2 version 2.28 [5] } \\
\hline & Network Size & $1500 \mathrm{~m} \times 300 \mathrm{~m}$ \\
\hline & Nodes & 25,50 \\
\hline & Initial Battery Power & 1500 Joules \\
\hline \multirow{2}{*}{ Physical Layer } & Signal Propagation Model & Two-ray ground reflection model \\
\hline & Transmission Range & $250 \mathrm{~m}$ \\
\hline \multirow{3}{*}{ MAC Layer } & \multicolumn{2}{|c|}{ IEEE $802.11[8]$} \\
\hline & Link Bandwidth & $2 \mathrm{Mbps}$ \\
\hline & Interface Queue & FIFO-based, Size 100 \\
\hline Routing Protocols & \multicolumn{2}{|c|}{ ABR [24], FORP [23], RABR [1] } \\
\hline \multirow{4}{*}{ Mobility Model } & \multicolumn{2}{|c|}{ Random-way point model [2] } \\
\hline & Minimum Node Speed & $0 \mathrm{~m} / \mathrm{s}$ \\
\hline & Maximum Node Speed $\left(v_{\max }\right)$ & $1,5,10,15,20$ and $30 \mathrm{~m} / \mathrm{s}$ \\
\hline & Pause Time & 0 Second \\
\hline \multirow{4}{*}{ Traffic Model } & \multicolumn{2}{|c|}{ Constant Bit Rate (CBR), UDP } \\
\hline & Number of Source-Destination $(s-d)$ Sessions & 15 and 30 \\
\hline & Data Packet Size & 512 bytes \\
\hline & Packet Sending Rate per $s-d$ Pair & 4 Packets/sec \\
\hline \multirow{4}{*}{ Energy Consumption Model } & Transmitting Power & $1.327 \mathrm{~W}$ \\
\hline & Receiving Power & $0.967 \mathrm{~W}$ \\
\hline & Idle Power & $0 \mathrm{~W}$ \\
\hline & Promiscuous Listening & No \\
\hline
\end{tabular}




\subsection{Physical, Data Link and MAC Layer Models}

The physical, data link and MAC layer models [3] are based on the multi-hop wireless network extension provided in ns-2. The MAC layer uses the distributed co-ordination function (DCF) of the IEEE Standard 802.11 [8] for wireless LANs. The radio model uses the standard channel bandwidth of 2 Mbps. The signal propagation model used is the two-ray ground reflection model [3]. The interface queue is used to queue both the routing and data packets sent by the routing layer until the MAC layer is able to transmit them. We use a FIFO-based interface queue of length 100 for all the three protocols. Higher priority is given to route discovery and route maintenance packets.

\subsection{Node Mobility Model}

The node mobility model used in all of our simulations is the Random Waypoint model [2], a widely used mobility model in MANET simulation studies. According to this model, each node starts moving from an arbitrary location to a randomly selected destination location at a speed uniformly distributed in the range $\left[v_{\min }, \ldots, v_{\max }\right]$. Once the destination is reached, the node may stop there for a certain time called the pause time and then continue to move by choosing a different target location and a different velocity. In this paper, we set $v_{\text {min }}$ $=0$ and each node chooses speed uniformly distributed between 0 and $v_{\max }$. The $v_{\max }$ values used are $1,5,10,15,20$ and $30 \mathrm{~m} / \mathrm{s}$. Pause time is 0 seconds. We categorize $v_{\max }$ values of 1 and $5 \mathrm{~m} / \mathrm{s}$ as low mobility, 10 and $15 \mathrm{~m} / \mathrm{s}$ as moderate mobility and 20 and $30 \mathrm{~m} / \mathrm{s}$ as high mobility scenarios.

\subsection{Traffic Model}

Traffic sources are continuous bit rate (CBR). The number of source-destination $(s-d)$ sessions used are 15 (low traffic load) and 30 (high traffic load). The starting times of these $s-d$ sessions is uniformly distributed between 0 to 100 seconds and each $s-d$ session once begun, lasts till the end of the simulation. Data packets are 512 bytes in size and the packet sending rate is 4 data packets/second. While distributing the source-destination roles for each node, we saw to it that a node does not end up as source of more than two sessions and also not as a destination for more than two sessions.

\subsection{Energy Consumption Model}

The energy consumption at a node in an ad hoc network can be divided into three categories: (1) Energy utilized for transmitting a message, (2) Energy utilized for receiving a message and (3) Energy utilized in idle state. In [12], it has been shown that in the presence of overhearing, no real optimization in the energy consumption or the node lifetime can be achieved. That is, the energy consumption at a node would be dominated by the energy lost when the node is in the idle state (also referred to as being in the promiscuous mode). Thus, in this paper, we do not consider the energy lost in the idle state and focus only on the energy consumed during the transmission and reception of messages (data packets, the MAC layer RTS-CTS packets and the periodic beacons), and energy consumed due to route discoveries. 
The initial battery charge at all nodes is 1500 Joules. We do not perform any power control. Irrespective of the physical length of a hop (i.e., the physical distance between two nodes forming a hop), we use the same fixed transmission power per hop. We model the energy consumed due to broadcast traffic and point-to-point traffic as linear functions of the packet transmission time, network density, transmission and reception powers per hop. A similar linear modeling for energy consumption has been used in [6][14].

\subsection{Beacon Messaging}

All the three protocols use beacon control messages (exchanged every one second) to let each node advertise its presence to its neighbors. With ABR, we will let each node to mention its current velocity in the beacon message to its neighbors. This will help a neighboring node to calculate the relative velocity between itself and the node sending the beacon message. In the case of RABR, we will let each node to periodically, for every one second, sample the signal strength of the last 10 beacons received from each of its neighbors. For FORP, we will let each node to send information about its location and current velocity in the beacons. Each node will also keep track of the previously advertised location of its neighbor nodes. This will help to determine the direction in which the neighbor node is moving.

\subsection{Performance Metrics}

Each data point in Figures 1 through 7 is an average of the data collected using 5 mobility trace files and 5 sets of randomly selected 15 and $30 s-d$ sessions. We study the following performance metrics for the three protocols when run under the specific scenarios listed in the caption for each of Figures 1 through 7.

(i) Number of route transitions - average of the number of route discoveries required for all the $s$ - $d$ sessions (refer Figure 1).

(ii) Hop count per route - average of the number of hops in the routes, time-averaged over all the $s$ - $d$ sessions (refer Figure 2).

(iii)End-to-end delay per packet - average of the delay incurred by all the packets that originate at the source and are delivered at the destination (refer Figure 3). The delay incurred by a packet includes all the possible delays - the buffering delay due to the route acquisition latency, the queuing at the interface queue to access the medium, transmission delay, propagation delay, and the retransmission delays due to the MAC layer collisions.

(iv)Packet delivery ratio - ratio of the number of packets delivered to the destination to those generated by the CBR sources (refer Figure 4).

(v) Energy Consumed per Packet - average of the energy consumed by all the packets that originate at the source and are delivered at the destination (refer Figure 5). We include the energy consumed due to transmission and reception of data packets, MAC layer packets and the energy consumed due to route discoveries.

(vi)Energy Consumed per Node - average of the energy consumed across all the nodes in the network (refer Figure 6). The energy consumed at a node includes the energy lost due to the 
transmission and reception of data packets, MAC layer packets, periodic beacons exchange, and the energy consumed due to route discoveries.

(vii)Standard Deviation of Energy Consumed per Node - Square root of the average of the squares of the difference between the energy consumed at each node and the average energy consumed per node (refer Figure 7). This metric is used to evaluate the fairness of node usage by each protocol. Ideally, the value of this metric should be zero to indicate that all nodes have been used fairly and no node is overused.

\subsection{Number of Route Transitions}

In all the figures related to the number of route transitions (refer Figure 1), we can see a clear ranking of the three protocols. FORP is better than RABR, which is better than ABR. This in turn indicates a ranking among the strategies to make use of the knowledge of the past and/or future topology changes. The strategy of making use of only the knowledge of past topology changes as in the case of ABR (e.g., the number of beacons exchanged between two nodes) cannot quite accurately predict the future. Even though two nodes may be moving in the vicinity of each other beyond the duration of the association threshold, we don't know whether they are moving towards each other or away from each other. While selecting stable paths, it is very essential to predict and use information about the residual lifetime of a link (i.e., how long two nodes will stay together as neighbors) rather than just use information about the link age (i.e., how long two nodes have stayed together as neighbors). This is what exactly being done by protocols RABR and FORP.

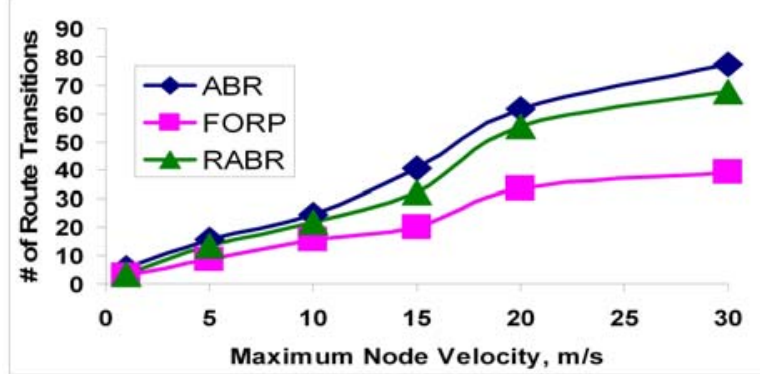

Figure 1.1: $15 s$ - $d$ Pairs, 25 Nodes

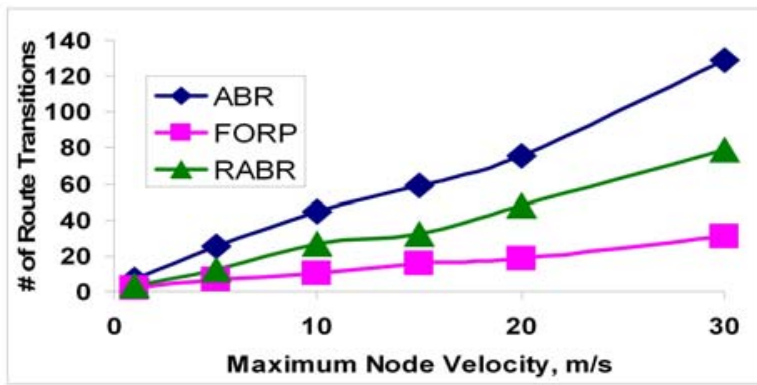

Figure 1.3: $15 s-d$ Pairs, 50 Nodes

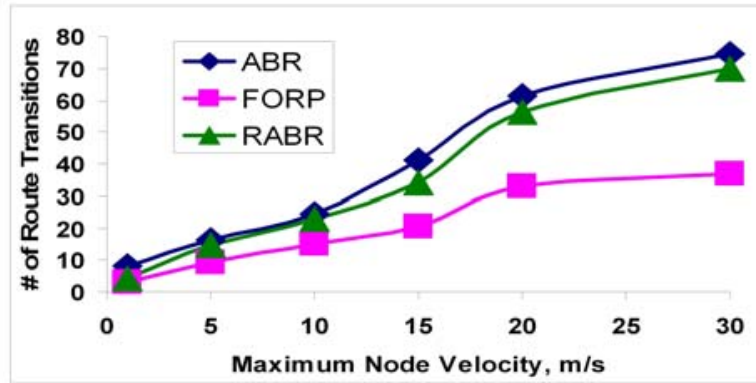

Figure 1.2: $30 s-d$ Pairs, 25 Nodes

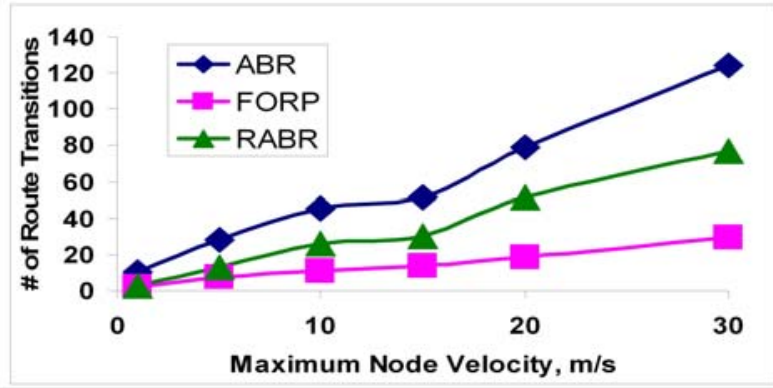

Figure 1.4: $30 s$ - $d$ Pairs, 50 Nodes

Figure 1: Average Number of Route Transitions for ABR, FORP and RABR 
Using location-update mechanisms to predict the lifetime of the links and hence the residual lifetime of the paths seems to be the most accurate strategy of finding stable paths. FORP completely forgets about the past and simply selects routes based on the prediction of the future topology changes. But in practical applications, it may not be quite easy to use GPS and other location-update mechanisms without significant loss in the battery reserves at the nodes. RABR does not require any expensive location-prediction mechanism like that of FORP and it quite effectively tries to predict the future based on the average change in the signal strength of the beacons exchanged among neighboring nodes. If the average change in the strength of the beacon signals exchanged in the recent past is negative, it indicates the two nodes have started to move away from each other as the strength of the beacon signals emanating from them is deteriorating with time. On the other hand, if the average change in the signal strength of the beacons exchanged in the recent past is positive, it indicates the two nodes have started to move towards each other. Once two nodes start moving towards each other, it is likely to take a reasonable amount of time for them to move away from each other. This is the fact that is being explored by RABR to find stable routes.

For a given node mobility, as the network density increases, the absolute number of route transitions for FORP either decreases or remains almost the same as observed for low network density. The protocol tries to make use of the increase in the available number of nodes and selects the path that will exist for the longest time from the current instant, but ends up with a $20-40 \%$ increase in the hop count. With increase in the hop count, it might be possible to conjecture a high probability of route failure. But the probability of a route failure very much depends on the probability of the failure of the constituent links too. If the probability of failure of each individual link gets reduced as network density increases, we could manage a relatively better stable path as long as the increase in the hop count is not large enough.

\subsection{Average Hop Count per Route}

With increase in the network density, we see that the stability of the routes found by FORP increases while the stability of the routes found by ABR decreases. This could be attributed to the following reasoning: At high densities, there are likely to be multiple candidate paths that have the maximum proportion of links with the threshold association stability. In such cases, ABR chooses the path with the minimum hop count among the candidate paths. As observed in [13], minimum hop count paths suffer under the edge effect problem, especially at high densities. The physical length of a hop is close to $70-80 \%$ of the transmission range of the nodes and hence the hops are highly vulnerable to fail in the near future. Note that, even though ABR selects the minimum hop path among the paths that have the maximum proportion of links with the threshold association stability, we do not know whether the constituent nodes of the links are moving away or towards each other. One or more pairs of neighboring nodes in a path are more likely to move away from each other so that the physical distance between the source and the destination is covered with a reduced number of hops in the route.

On the other hand, FORP tries to make use of the increase in the available number of 
nodes and selects the path that will exist for the longest time from the current instant. As a result, with increase in network density, FORP finds relatively better stable routes and the tradeoff is an increase in the hop count per route. The physical length of the hops chosen by FORP is only $50-60 \%$ of the transmission range of the nodes and hence a link is less susceptible to break away in the near future. RABR is the least sensitive to the number of route transitions and hop count with respect to network density. The reason is the affinity values of links are more diverse and is not a single value like the threshold association stability. Hence, it is less likely there would be two or more paths with the same bottleneck affinity value and the tie would be broken using a minimum hop path. The decision of whether two nodes are moving towards each other or moving away from each other is least affected by the number of nodes in the network.

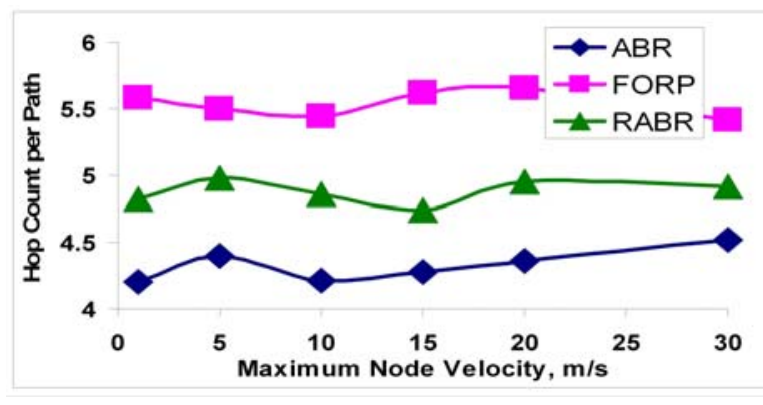

Figure 2.1: $15 s-d$ Pairs, 25 Nodes

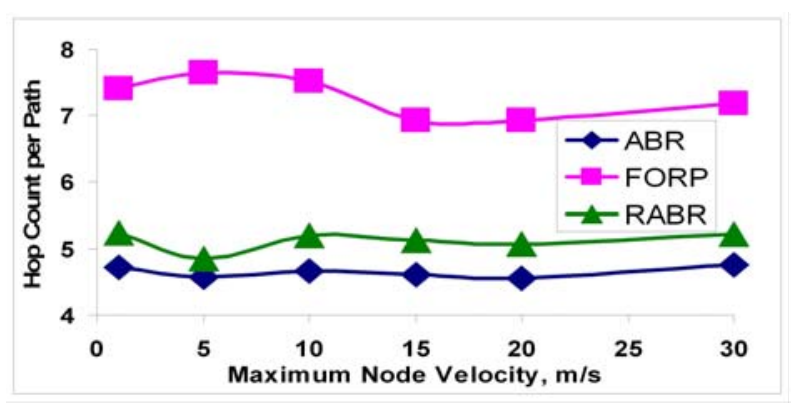

Figure 2.3: $15 s-d$ Pairs, 50 Nodes

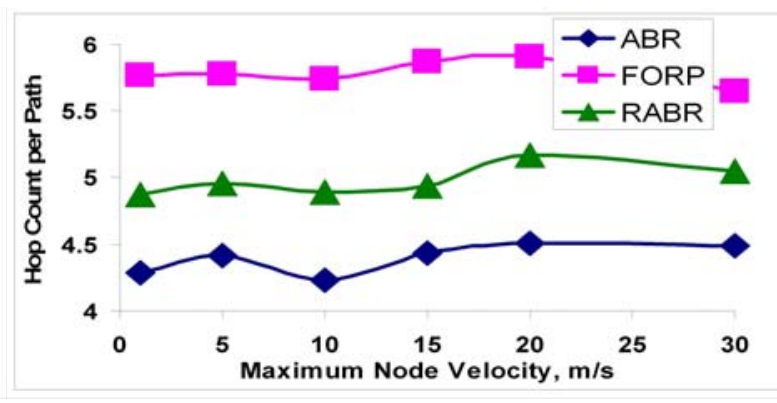

Figure 2.2: $30 s-d$ Pairs, 25 Nodes

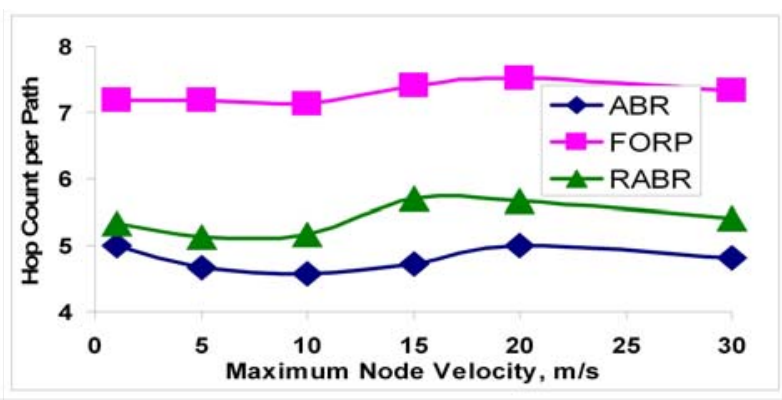

Figure 2.4: $30 s-d$ Pairs, 50 Nodes

Figure 2: Average Hop Count per Route for ABR, FORP and RABR

\subsection{End-to-End Delay per Data Packet}

At low and moderate mobility, ABR and RABR incur lower end-to-end delay per packet compared to FORP. This could be attributed to the higher route relaying load on the nodes in the case of FORP. Especially, when the number of $s-d$ sessions is 30, packets transferred through FORP routes incur significant delays due to MAC layer contention, and queuing before transmission. Thus, we see a stability-delay tradeoff at low and moderate velocities. Larger the desire for stability, larger is the end-to-end delay per packet that would be incurred. RABR reduces this tradeoff to a certain extent by maintaining the hop count within a reasonable limit and achieves stability in its routes. RABR achieves delay close to ABR at low and moderate velocities and at the same time achieves stability close to that of FORP. In 
other words, RABR achieves a right balance between the route relaying load per node and the route discovery latency. On the other hand, the buffering delay due to the route acquisition latency plays a significant role in increasing the delay of ABR routes and to a certain extent the RABR routes at high velocity. The delay incurred in having multiple intermediate nodes in the FORP routes is compensated by the route acquisition delay of ABR routes. At high traffic load and network density, RABR routes incur the lowest delay.

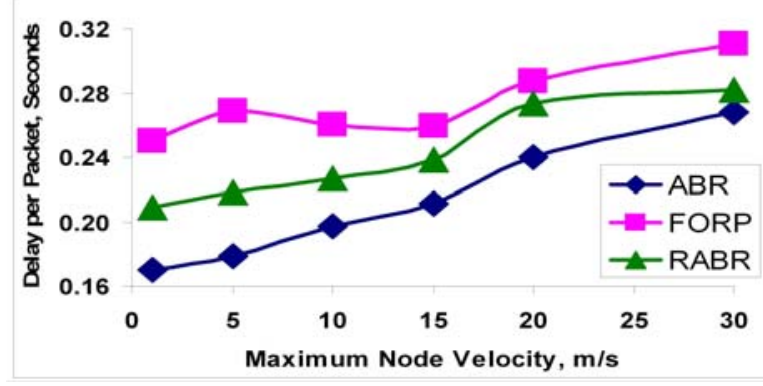

Figure 3.1: $15 s-d$ Pairs, 25 Nodes

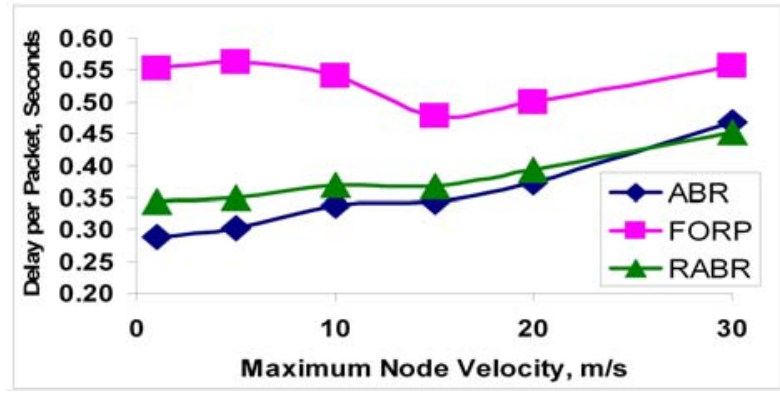

Figure 3.3: $15 s-d$ Pairs, 50 Nodes

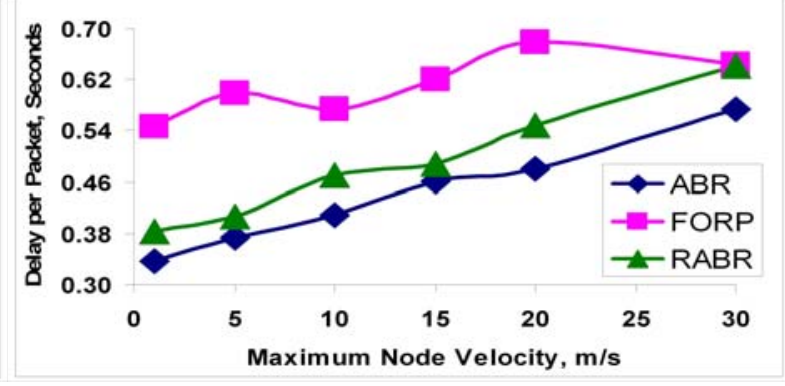

Figure 3.2: $30 s-d$ Pairs, 25 Nodes

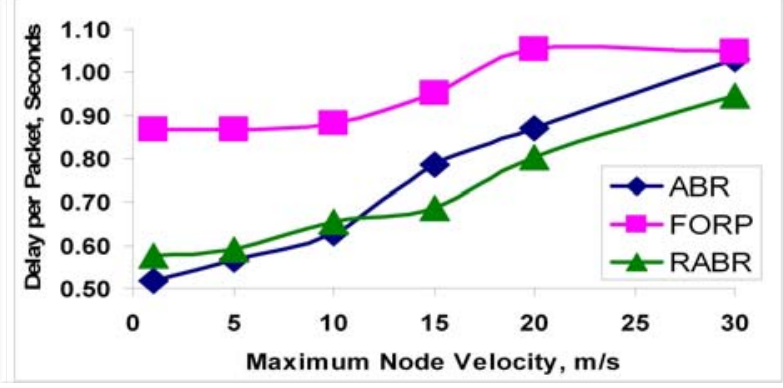

Figure 3.4: $30 s-d$ Pairs, 50 Nodes

Figure 3: Average End-to-End Delay per Data Packet for ABR, FORP and RABR

It is more obvious to see that for a given network density, higher the number of simultaneous $s$ - $d$ sessions in the network, higher will be the end-to-end delay per packet. On the other hand, the number of MAC layer collisions increases with increase in the network density and the number of $s$ - $d$ sessions. Also, the beacon processing delay at the nodes, which increases with increase in network density, affects the end-to-end delay per data packet. The effect could be seen in the relatively higher delays incurred per packet in the case of 50 nodes with $30 s-d$ sessions compared to the case of 25 nodes with $30 s-d$ sessions, even though in the latter case, each node ends up as a source and/or destination for more than one $s$ - $d$ session.

\subsection{Packet Delivery Ratio}

In the wake of a route failure, $A B R$ tries to do a local route repair before going for a global route discovery. During this phase, data packets need to be buffered at intermediate nodes. But local repair is successful only at low velocities. As the velocity increases, local route repairs become unsuccessful and global route discovery needs to be initiated. The data 
packets stored in the buffer of intermediate nodes timeout and get dropped. As a result, the packet delivery ratio, which is the ratio of the number of data packets successfully delivered to the destination to that of the total number of data packets originating at the source, decreases at a faster rate compared to that of the relatively more stable FORP and RABR. In the case of RABR, an intermediate node immediately notifies the source node using a ROUTE-ERROR packet upon failure of a data transmission. The inherent stability of the routes chosen by RABR also helps in reducing the number of route failures. FORP routes are highly stable and hence most of the data packets that originate at the source are delivered to the destination without being retransmitted or being dropped. Note that the queue sizes we use are sufficiently large to avoid any significant congestion in the network.

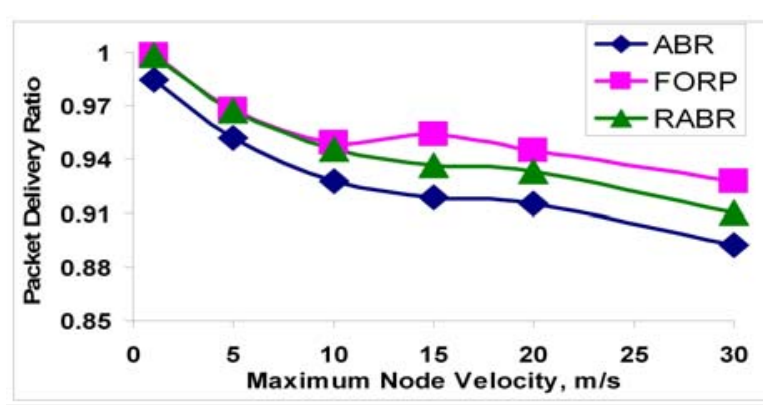

Figure 4.1: $15 s-d$ Pairs, 25 Nodes

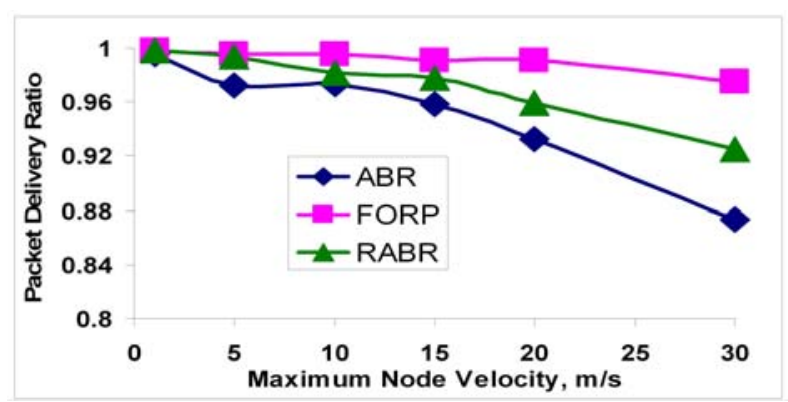

Figure 4.3: $15 s-d$ Pairs, 50 Nodes

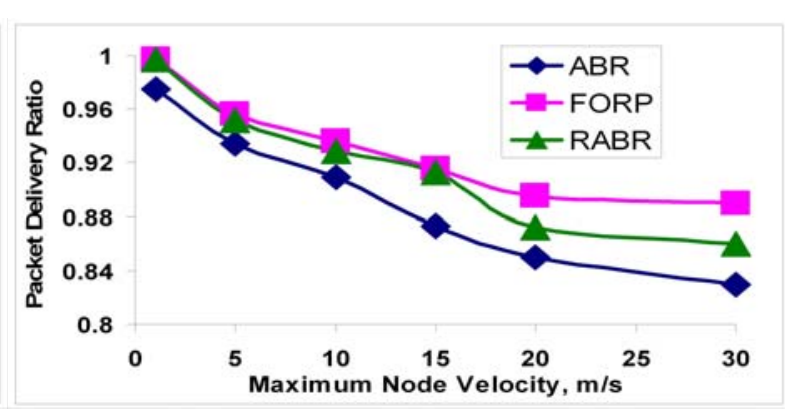

Figure 4.2: $30 s$ - $d$ Pairs, 25 Nodes

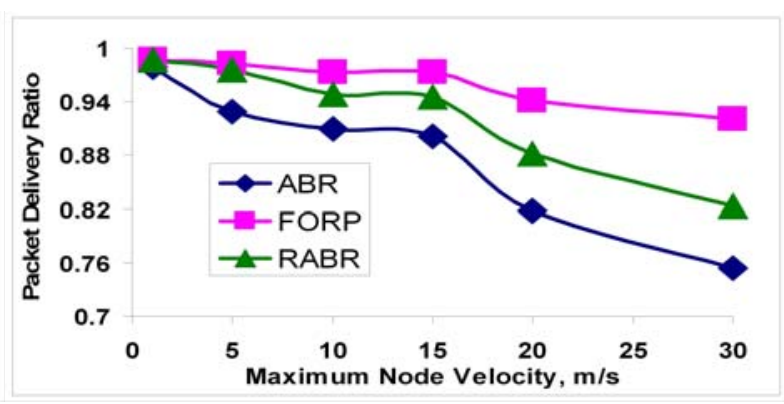

Figure 4.4: $30 s$ - $d$ Pairs, 50 Nodes

Figure 4: Average Packet Delivery Ratio for ABR, FORP and RABR

\subsection{Energy Consumption}

The energy spent per packet is the sum of the energies consumed during transmission of the data packet (including the MAC layer packet exchanges) and the route discoveries divided by the total number of packets generated by all the CBR sources. The energy consumed per packet is predominantly decided by the number of hops per route and the network density. Higher the number of hops, higher the energy consumed per packet for the protocol. This is understandable as we do not employ any power control to take into account the physical length of a hop. To understand the impact of network density, note that we take into consideration the energy spent by each node in receiving the MAC layer RTS-CTS packets. For a given hop count, higher the network density, higher the amount of energy spent by each node in receiving the MAC layer packets exchanged in a neighborhood. As FORP 


\section{Macrothink

incurs the maximum number of hops per route, the protocol incurs higher energy consumption per packet compared to that of RABR and ABR. At high network density, FORP also takes the liberty of going through relatively more nodes such that the lifetime of a path can be maximized. As a result of all these factors, the energy spent per packet at high network density in a FORP route is twice the amount of energy spent at low network density. At high network density, the hop count of the routes chosen by RABR and ABR protocols is $40 \%$ less than that of FORP routes. Thus, the energy consumed per packet for RABR and ABR routes at high network density is only $50 \%$ more than that consumed at less network density.

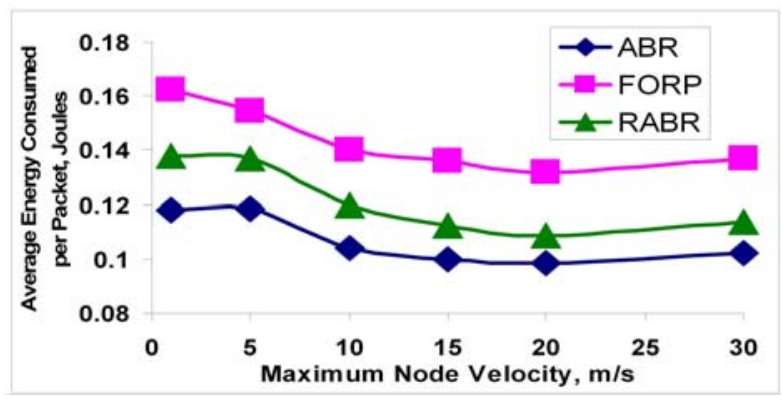

Figure 5.1: $15 s-d$ Pairs, 25 Nodes

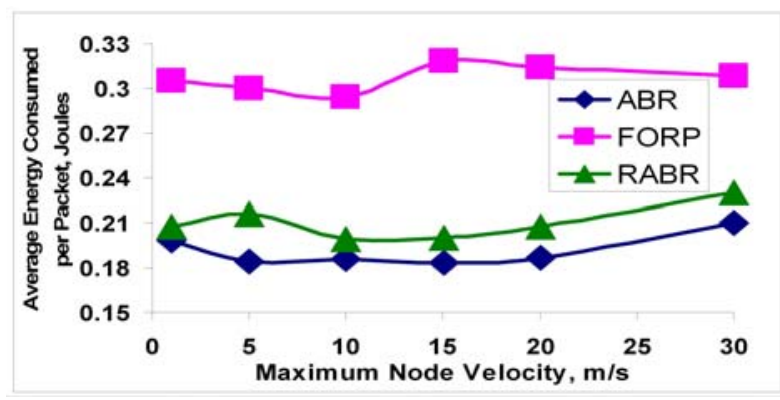

Figure 5.3: $15 s-d$ Pairs, 50 Nodes

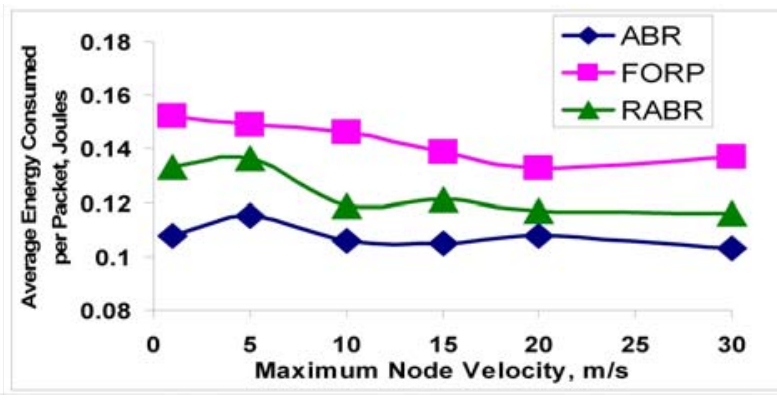

Figure 5.2: $30 s$ - $d$ Pairs, 25 Nodes

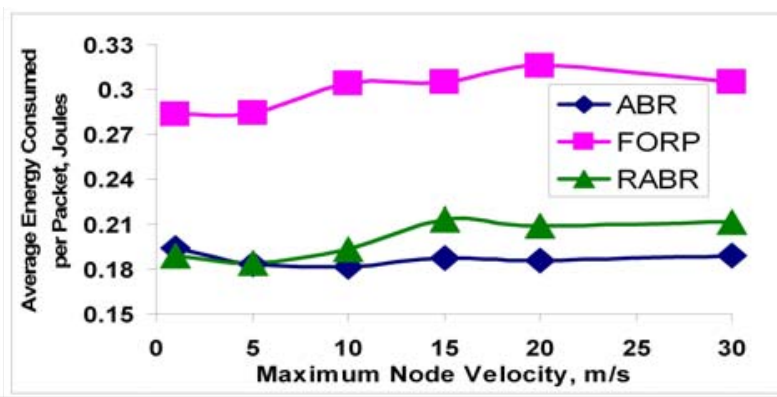

Figure 5.4: $30 s-d$ Pairs, 50 Nodes

Figure 5: Average Energy Consumed per Data Packet for ABR, FORP and RABR

At low network density, as the node velocity increases, protocols tend to select paths with a fewer number of hops compared to the paths chosen when the network is less mobile. This is because the average lifetime of a path is limited at high node mobility, and there is no significant increase in the lifetime of the paths that have a larger hop count. Hence, the protocols end up choosing paths with lower hop count and limited lifetime. In fact, paths with larger hop count are more likely to break soon, as the nodes constituting a hop can drift away any time in the near future as the topology keeps changing dynamically in the near future. As a result, the amount of energy spent in route discoveries does make an impact on the overall energy consumption at high node mobility and high network density, especially for ABR. However, at low network density, the amount of energy spent in route discoveries does not significantly affect the overall energy consumption. This is because the number of nodes that receive the ROUTE REQUEST packets per broadcast is less at low network density. 

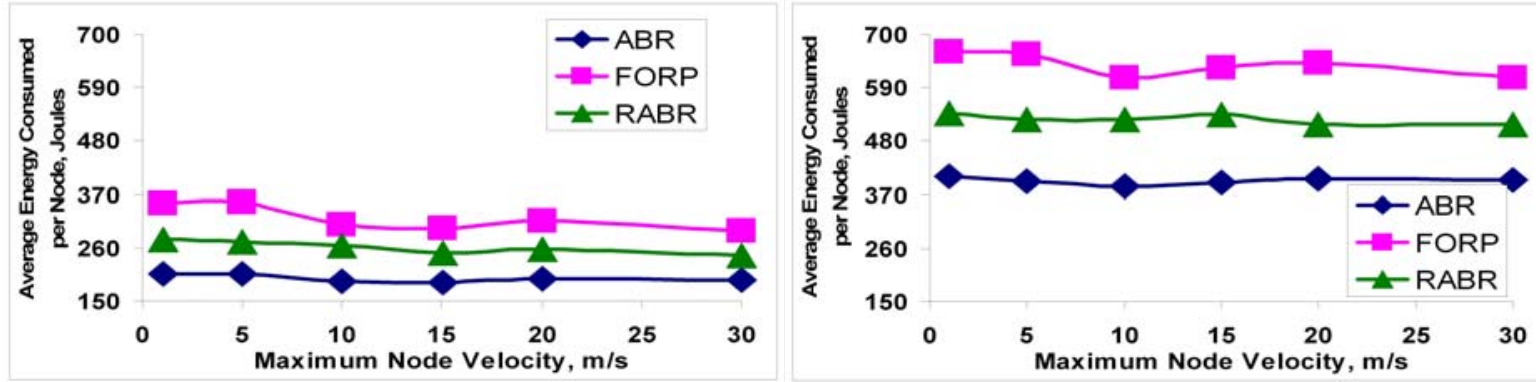

Figure 6.1: $15 s$ - $d$ Pairs, 25 Nodes

Figure 6.2: $30 s-d$ Pairs, 25 Nodes

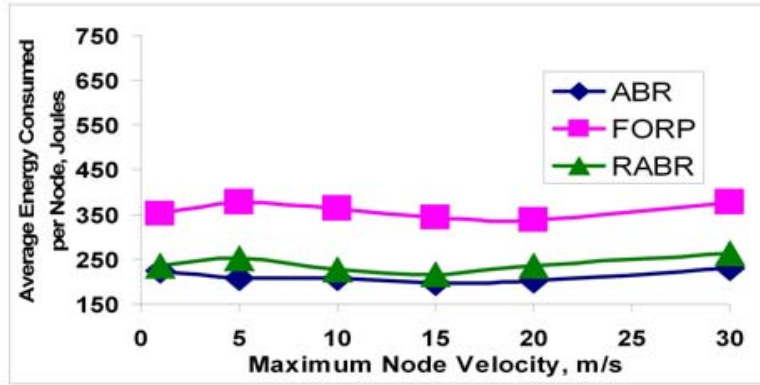

Figure 6.3: $15 s$ - $d$ Pairs, 50 Nodes

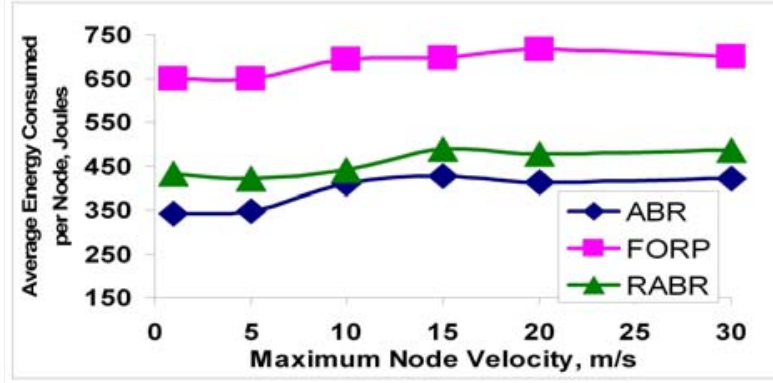

Figure 6.4: $30 s-d$ Pairs, 50 Nodes

Figure 6: Average Energy Consumed per Node for ABR, FORP and RABR

\subsection{Fairness of Node Usage}

The fairness of node usage of the three protocols is studied by evaluating the average energy spent per node and the standard deviation of the energy spent per node. For a given network density and node mobility, it is obvious that higher the number of source-destination pairs, higher the amount of energy spent per node. An interesting observation is that for a given traffic intensity, the standard deviation of the energy consumed per node at high network density decreases by $50 \%$ (at low mobility) to $20 \%$ (at high mobility) compared to that at low network density. This is because of the tendency of the protocols to increase the stability of routes by making use of the increase in the available number of nodes. This helps to divert the traffic to more nodes, share the offered load equally and thus increase the fairness of node usage. Thereby, the average energy consumed per node is also kept within reasonable limits and is not allowed to increase significantly in comparison to that incurred at low network density. Also, one could observe that for a given network density, the standard deviation of the energy consumed per node decreases with increase in the node mobility. This is because routes get distributed more evenly when the network topology changes dynamically. But, the average energy consumed per node does not significantly differ with node mobility, especially at low network density.

The average energy consumed per node is the highest in the case of FORP. This is because of the higher number of hops per path compared to that of ABR and RABR. For a given node mobility, irrespective of the network densities, the standard deviation in the energy consumed per node remains low when the offered traffic load (i.e., the number of $s-d$ 
sessions) is low. But, with increase in the offered traffic load, the standard deviation in the energy usage per node increases. This is because the protocols find stable routes for relatively higher number of $s-d$ sessions. As long as the sequence of topology changes remains the same (i.e., for a fixed node mobility), the number of nodes that are used as part of the stable routes does not increase significantly with increase in the offered traffic load. Only the nodes that can be part of stable routes are exhausted to a greater extent. This is also the reason that the standard deviation of energy usage per node is high for FORP compared to that of ABR and RABR.

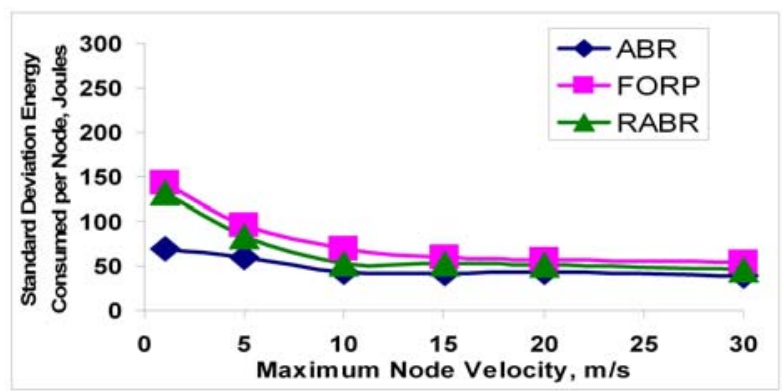

Figure 7.1: $15 s-d$ Pairs, 25 Nodes

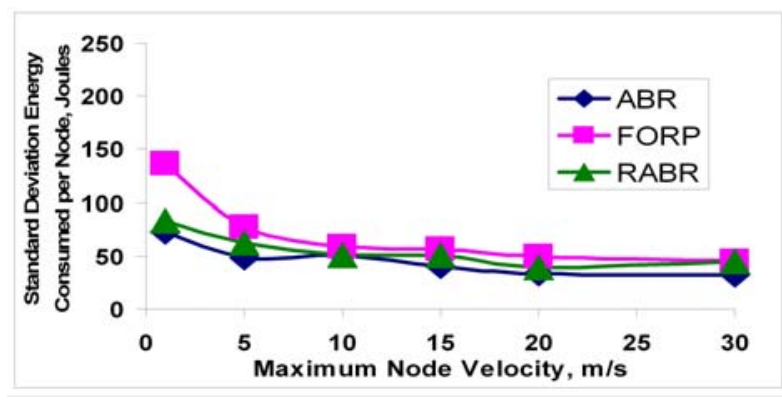

Figure 7.3: $15 s$ - $d$ Pairs, 50 Nodes

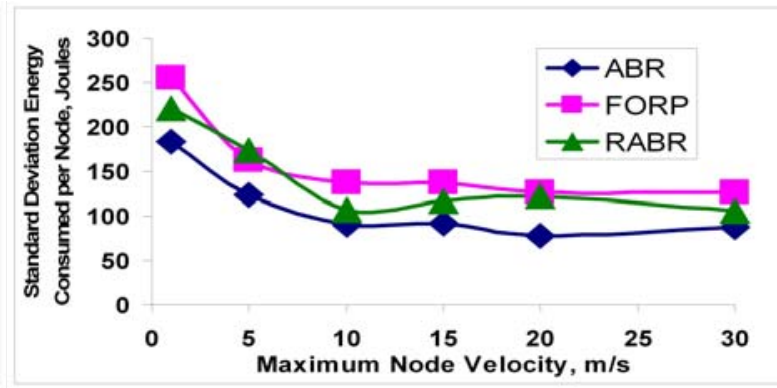

Figure 7.2: $30 s-d$ Pairs, 25 Nodes

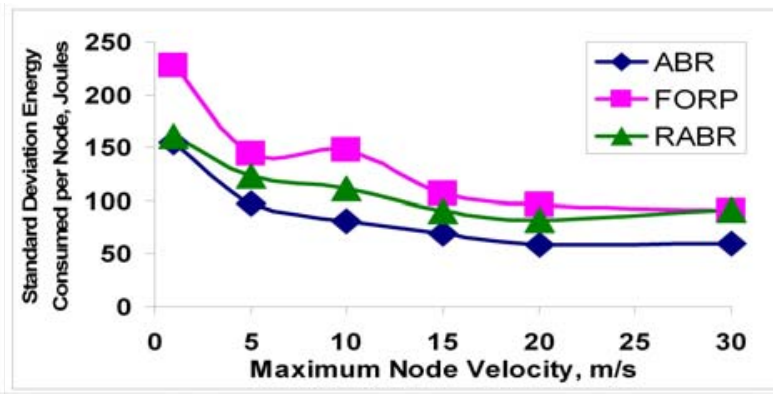

Figure 7.4: $30 s-d$ Pairs, 50 Nodes

Figure 7: Standard Deviation of Energy Consumed per Node for ABR, FORP and RABR

\section{Conclusions and Future Work}

We could draw two significant conclusions. One, we see a stability-delay-energy consumption tradeoff among the stability-oriented routing protocols for MANETs. Higher the stability, higher is the end-to-end delay and more likely higher energy consumption per packet. RABR reduces this tradeoff to a certain extent by maintaining a proper balance between the route propagation load and route stability. FORP routes are the most stable of the three protocols but also incur the highest delay and energy consumption per packet because of the increase in the number of intermediate nodes that forward a packet. ABR routes incur the lowest delay and energy consumption at low and moderate velocities, but are also the least stable of the three protocols. At high velocities, the route acquisition latency of ABR routes significantly increases the overall delay per packet as local route repairs fail and global route discovery has to be frequently initiated. ABR is slightly better than RABR in terms of energy consumption per packet among the three protocols at high node mobility. Nevertheless, 
for real-time applications, RABR would be more suitable at very high velocities and especially at high network densities, as it is relatively more stable than ABR, incurs lower delay and incurs only slightly higher energy consumption. As the network density and node mobility increases, we see RABR to be the best protocol that can neutralize the stability-delay-energy consumption tradeoff.

The second conclusion that could be drawn from this paper is that strategies that are exclusively based on the knowledge of the past topology changes are not sufficient to select highly stable routes. It is not enough to predict the future of a link based on how long the link existed so far. We need to estimate or predict the residual lifetime of a link (i.e., how long a link will exist from the current time instant). RABR does this by estimating the average change in the signal strength in the near past. FORP predicts the lifetime of a link using location and mobility information available through beacon exchange. The mobility prediction mechanism of FORP yields a more accurate measure of the lifetime of a link than the signal strength based mechanism of RABR. The strength of RABR is that it does not require any expensive location-update mechanisms like that of FORP to predict the lifetime of a link. Note that GPS and other location-update mechanisms consume the battery charge of the nodes. The strategy used by RABR is a mix of both the past knowledge and future prediction. The signal strength of the beacons exchanged in the past is used as well as the average change in the signal strength to capture information about the future.

We make the following recommendations: For real-time applications that are most often deployed in energy constrained and disaster relief environments, delay per packet may be the most important metric and out-of-order packet arrival can be tolerated to a certain extent. Hence, ABR is recommended for such environments. For applications that require minimal use of location-update mechanisms and other external resources, fairness of node usage, and that all the three metrics, viz., delay, route stability and energy consumption are to be given equal importance, then RABR is the best choice. For some Quality of Service applications like video conferencing and electronic classrooms (say, using a network of laptops that could be continuously charged by being plugged to an electric outlet), the primary concern would be to ensure a continuous delivery of packets without much jitter. The energy reserves may be available in plenty and delay may not be a primary concern as long as it is within a certain bound. In such situations, it would be worth opting for FORP and other protocols that can predict highly stable routes based on the location-update mechanisms and the prediction of future topology changes. In a recent work, we have proposed a beaconless node velocity based stable path routing protocol [18] that uses a Min-Max approach to determine the path with the smallest bottleneck velocity, where the bottleneck velocity of a path is the maximum of the velocities of the intermediate nodes of the path. In another related work [19], we have proposed a multicast extension for the FORP and NVSP to determine stable multicast trees.

As part of future work, we will study the performance of the NVSP protocol along with the three routing protocols - ABR, FORP and RABR - under different mobility models [4]. The mobility models that would be explored include the Random Waypoint model, the Random Walk model, the Gauss-Markov model, the Reference Point Group Mobility (RPGM) model, the City Section model and the Manhattan model. 


\section{References}

[1] S. Agarwal, A. Ahuja, J. P. Singh and R. Shorey, "Route-Life Time Assessment Based Routing Protocol for Mobile Ad Hoc Networks," Proceedings of IEEE ICC, June 2000.

[2] C. Bettstetter, H. Hartenstein and X. Perez-Costa, "Stochastic Properties of the Random-Way Point Mobility Model," Wireless Networks, pp. 555 - 567, Vol. 10, No. 5, September 2004.

[3] J. Broch, D. A. Maltz, D. B. Johnson, Y. C. Hu and J. Jetcheva, "A Performance Comparison of Multi-hop Wireless Ad hoc Network Routing Protocols," Proceedings of MobiCom, pp. 85 - 97, October 1998.

[4] T. Camp, J. Boleng and V. Davies, "A Survey of Mobility Models for Ad Hoc Network Research," Wireless Communication and Mobile Computing, Vol. 2, No. 5, pp. 483-502, September 2002.

[5] K. Fall, K. Varadhan, "The ns Manual," The VINT Project, A Collaboration between researchers at UC Berkeley, LBL, USC/ISI and Xerox PARC.

[6] L. M. Feeney, "An energy-consumption model for performance analysis of routing protocols for mobile ad hoc networks," Journal of Mobile Networks and Applications Vol. 3, No. 6, pp. 239 - 249, June 2001.

[7] M. Gerharz, C de Waal, P. Martini and P. James, "Strategies for Finding Stable Paths in Mobile Wireless Ad Hoc Networks," Proceedings of the $28^{\text {th }}$ IEEE International Conference on Local Computer Networks, pp. 130-139, October 2003.

[8] IEEE Standards Department, Wireless LAN Medium Access Control (MAC) and Physical Layer (PHY) Specifications, IEEE Standard 802.11-1997, 1997.

[9] P. Johansson, T. Larson, N. Hedman, B. Mielczarek and M. DegerMark, "Scenario-based Performance Analysis of Routing Protocols for Mobile Ad hoc Networks," in Proceedings of MOBICOM, 1999.

[10] D. B. Johnson, D. A. Maltz and J. Broch, "DSR: The Dynamic Source Routing Protocol for Multi-hop Wireless Ad hoc Networks," Ad hoc Networking, edited by Charles E. Perkins, Chapter 5, pp. 139 - 172, Addison Wesley, 2001.

[11] Kaplan ED (ed.), Understanding the GPS: Principles and Applications, Artech House: Boston, MA; 1996.

[12] D. Kim, J.J. Garcia-Luna-Aceves, K. Obraczka, J-C. Cano, and P. Manzoni, "Routing Mechanisms for Mobile Ad Hoc Networks based on the Energy Drain Rate," IEEE Transactions on Mobile Computing, Vol. 2, No. 2, pp. 161-173, April-June 2003.

[13] G. Lim, K. Shin, S. Lee, H. Yoon and J. S. Ma, "Link Stability and Route Lifetime in Ad hoc Wireless Networks," Proceedings of International Conference on Parallel Processing Workshops, pp. 116 - 123, August 2002.

[14] N. Meghanathan and A. Farago, "Comparison of Routing Strategies for Minimizing Energy Consumption in Mobile Ad Hoc Networks," Proceedings of $4^{\text {th }}$ Asian International Mobile Computing Conference, 2006.

[15] N. Meghanathan and A. Farago, "Survey and Taxonomy of 55 Unicast Routing Protocols for Mobile Ad Hoc Networks," International Journal of Applications of Graph Theory in Wireless Ad hoc Networks and Sensor Networks, Vol. 1, No. 1, pp. 1 - 21, December 2009.

[16] N. Meghanathan, "Comparison of Stable Path Selection Strategies for Mobile Ad Hoc 


\section{Macrothink}

Networks," Proceedings of the $5^{\text {th }}$ IEEE International Conference on Networking, April 2006. [17] N. Meghanathan and A. Farago, "An Efficient Algorithm for the Optimal Number of Route Transitions in Mobile Ad Hoc Networks," IEEE International Conference on Wireless and Mobile Computing, Networking and Communications, Vol. 3, pp. 41 - 48, Aug. 2005.

[18] N. Meghanathan, "A Beaconless Node Velocity-based Stable Path Routing Protocol for Mobile Ad hoc Networks," Proceedings of the IEEE Sarnoff Symposium, pp. 9-13, Princeton, NJ, March 30-April 1, 2009.

[19] N. Meghanathan, D. Thomas and E. S. Addison, "Multicast Extensions to the Flow-Oriented Routing Protocol and Node Velocity-based Stable Path Routing Protocol for Mobile Ad hoc Networks," Proceedings of the 1st IEEE Workshop on Mobile Computing and Networking Technologies, St. Petersburg, Russia, October 12-14, 2009.

[20] V. D. Park and M. S. Corson, "A Highly Adaptive Distributed Routing Algorithm for Mobile Wireless Networks," Proceedings of IEEE INFOCOM, pp. 1405 - 1413, April 1997.

[21] C. E. Perkins and E. M. Royer, "Ad hoc On-demand Distance Vector Routing," Proceedings of the $2^{\text {nd }}$ Annual IEEE International Workshop on Mobile Computing Systems and Applications, pp. 90 - 100, February 1999.

[22] C. E. Perkins and P. Bhagwat, "Highly Dynamic Destination Sequenced Distance Vector Routing for Mobile Computers," ACM SIGCOMM, pp. 234 - 244, October 1994.

[23] W. Su, S-J Lee and M. Gerla, "Mobility Prediction and Routing in Ad Hoc Wireless Networks," International Journal of Network Management, vol. 11, no. 1, pp. 3-30, Jan-Feb. 2001.

[24] C-K. Toh, "Associativity-Based Routing for Ad hoc Mobile Networks," IEEE Personal Communications, Vol. 4, No. 2, pp. 103 - 139, March 1997.

\section{Copyright Disclaimer}

Copyright reserved by the author(s).

This article is an open-access article distributed under the terms and conditions of the Creative Commons Attribution license (http://creativecommons.org/licenses/by/3.0/). 